Ekemini D. Akpan, Sizwe J. Zamisa and Bernard Omondi*

\title{
Crystal structure of 2,4-pentanedione bis(2,4-dinitrophenylhydrazone), $\mathrm{C}_{17} \mathrm{H}_{16} \mathrm{~N}_{8} \mathrm{O}_{8}$
}

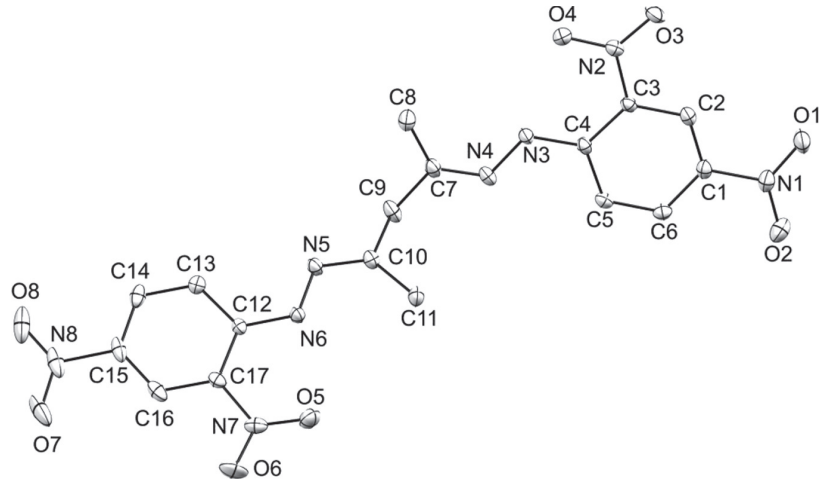

https://doi.org/10.1515/ncrs-2018-0509

Received November 19, 2018; accepted February 14, 2019; available online March 15, 2019

\section{Abstract}

$\mathrm{C}_{17} \mathrm{H}_{16} \mathrm{~N}_{8} \mathrm{O}_{8}$, orthorhombic, Pna2 $2_{1}$ (no. 33), $a=13.2406(3) \AA$, $b=21.0883(5) \AA, c=7.1364(2) \AA, V=1992.64(9) \AA^{3}, Z=4$, $R_{\mathrm{gt}}(F)=0.0285, w R_{\mathrm{ref}}\left(F^{2}\right)=0.0802, T=173(2) \mathrm{K}$.

CCDC no.: 1897170

The molecular structure is shown in the figure. Table 1 contains crystallographic data and Table 2 contains the list of the atoms including atomic coordinates and displacement parameters.

\section{Source of material}

The title compound was prepared adding of 2,4-pentadione (4.875 g, $0.04875 \mathrm{~mol}$ ) into a $100 \mathrm{~mL}$ round-bottom flask containing 2,4-dinitrophenylhydrazine (9.659 g, $0.04875 \mathrm{~mol}$ ). A brick red colour was observed and the contents of the flask were refluxed with continuous stirring overnight. Two layers were observed in the flask; an upper colourless layer and a lower red coloured layer. After the reflux, the flask was left to stand for two hours in an ice-water bath. A brown-orange

*Corresponding author: Bernard Omondi, University of KwaZulu-Natal, School of Chemistry and Physics, PO Box X01, Pietermaritzburg Campus, Scottsville, Pietermaritzburg 3209, South Africa, e-mail: owaga@ukzn.ac.za

Ekemini D. Akpan and Sizwe J. Zamisa: University of KwaZuluNatal, School of Chemistry and Physics, PO Box X54001, Westville Campus, Westville, 4000, Durban, South Africa
Table 1: Data collection and handling.

\begin{tabular}{ll}
\hline Crystal: & Needles, orange \\
Size: & $0.56 \times 0.29 \times 0.16 \mathrm{~mm}$ \\
Wavelength: & Mo $K \alpha$ radiation $(0.71073 \AA)$ \\
$\mu$ : & $0.13 \mathrm{~mm}^{-1}$ \\
Diffractometer, scan mode: & Bruker SMART, 0.5 $\varphi$ and $\omega$-scans \\
$\theta_{\text {max }}$, completeness: & $28.4^{\circ},>99 \%$ \\
$N(h k l)_{\text {measured }}, N(h k l)_{\text {unique }}, R_{\text {int }}:$ & $47896,4645,0.021$ \\
Criterion for $I_{\text {obs }}, N(h k l)_{\text {gt }}:$ & $I_{\text {obs }}>2 \sigma\left(I_{\text {obs }}\right), 4474$ \\
$N(\text { param })_{\text {refined }}:$ & 302 \\
Programs: & Bruker programs [1], SHELX [2], \\
& WinGX and ORTEP [3]
\end{tabular}

Table 2: Fractional atomic coordinates and isotropic or equivalent isotropic displacement parameters $\left(\AA^{2}\right)$.

\begin{tabular}{lrrrr}
\hline Atom & $\boldsymbol{x}$ & $\boldsymbol{y}$ & $\boldsymbol{z}$ & $\boldsymbol{U}_{\text {iso }}{ }^{*} \boldsymbol{U}_{\text {eq }}$ \\
\hline O1 & $0.12874(11)$ & $0.96896(6)$ & $0.1818(2)$ & $0.0338(3)$ \\
O2 & $0.27712(11)$ & $0.94063(7)$ & $0.0837(2)$ & $0.0356(3)$ \\
O3 & $-0.10620(10)$ & $0.81460(6)$ & $0.3784(2)$ & $0.0292(3)$ \\
O4 & $-0.08118(9)$ & $0.71373(6)$ & $0.34241(19)$ & $0.0243(3)$ \\
O5 & $0.53844(10)$ & $0.40681(6)$ & $0.3334(2)$ & $0.0324(3)$ \\
O6 & $0.61629(11)$ & $0.31851(8)$ & $0.3831(3)$ & $0.0403(4)$ \\
O7 & $0.48678(15)$ & $0.12102(7)$ & $0.2014(3)$ & $0.0532(5)$ \\
O8 & $0.33548(16)$ & $0.11237(7)$ & $0.0870(2)$ & $0.0488(5)$ \\
N1 & $0.19170(12)$ & $0.92861(7)$ & $0.1406(2)$ & $0.0255(3)$ \\
N2 & $-0.05413(10)$ & $0.76982(6)$ & $0.3247(2)$ & $0.0197(3)$ \\
N3 & $0.08197(10)$ & $0.67142(6)$ & $0.1768(2)$ & $0.0193(3)$ \\
H3 & 0.027855 & 0.658065 & 0.236496 & $0.023^{*}$ \\
N4 & $0.14432(11)$ & $0.62943(6)$ & $0.0822(2)$ & $0.0208(3)$ \\
N5 & $0.27611(11)$ & $0.43459(6)$ & $0.0947(2)$ & $0.0201(3)$ \\
N6 & $0.35752(10)$ & $0.40773(6)$ & $0.1875(2)$ & $0.0184(3)$ \\
H6A & 0.400833 & 0.431756 & 0.248438 & $0.022^{*}$ \\
N7 & $0.54301(11)$ & $0.34860(8)$ & $0.3252(2)$ & $0.0246(3)$ \\
N8 & $0.40548(16)$ & $0.14355(8)$ & $0.1523(3)$ & $0.0376(4)$ \\
C1 & $0.16322(12)$ & $0.86172(7)$ & $0.1568(2)$ & $0.0202(3)$ \\
C2 & $0.07121(13)$ & $0.84650(7)$ & $0.2311(2)$ & $0.0190(3)$ \\
H2 & 0.027103 & 0.878780 & 0.274979 & $0.023^{*}$ \\
C3 & $0.04309(11)$ & $0.78289(7)$ & $0.2414(2)$ & $0.0168(3)$ \\
C4 & $0.10707(12)$ & $0.73369(7)$ & $0.1743(2)$ & $0.0168(3)$ \\
C5 & $0.20221(11)$ & $0.75260(8)$ & $0.1012(2)$ & $0.0189(3)$ \\
H5 & 0.247811 & 0.721124 & 0.057483 & $0.023^{*}$ \\
C6 & $0.22971(12)$ & $0.81499(8)$ & $0.0924(3)$ & $0.0203(3)$ \\
H6 & 0.293690 & 0.826581 & 0.042831 & $0.024^{*}$ \\
C7 & $0.12319(12)$ & $0.57035(8)$ & $0.0988(3)$ & $0.0217(3)$ \\
C8 & $0.03931(14)$ & $0.54271(8)$ & $0.2137(3)$ & $0.0307(4)$ \\
H8A & -0.025750 & 0.553498 & 0.156511 & $0.046^{*}$ \\
H8B & 0.042182 & 0.560048 & 0.340977 & $0.046^{*}$ \\
H8C & 0.046654 & 0.496496 & 0.218707 & $0.046^{*}$ \\
& & & &
\end{tabular}

This work is licensed under the Creative Commons Attribution 4.0 
Table 2 (continued)

\begin{tabular}{lrrrr}
\hline Atom & $\boldsymbol{x}$ & $\boldsymbol{y}$ & $\boldsymbol{z}$ & $\boldsymbol{U}_{\text {iso }}{ }^{*} \boldsymbol{U}_{\text {eq }}$ \\
\hline C9 & $0.19058(15)$ & $0.52658(8)$ & $-0.0126(3)$ & $0.0257(4)$ \\
H9A & 0.223207 & 0.551078 & -0.114245 & $0.031^{*}$ \\
H9B & 0.148521 & 0.493262 & -0.071385 & $0.031^{*}$ \\
C10 & $0.27151(12)$ & $0.49534(8)$ & $0.1050(3)$ & $0.0200(3)$ \\
C11 & $0.34250(14)$ & $0.53693(8)$ & $0.2130(3)$ & $0.0258(4)$ \\
H11A & 0.412005 & 0.528672 & 0.172635 & $0.039^{*}$ \\
H11B & 0.336122 & 0.527790 & 0.347159 & $0.039^{*}$ \\
H11C & 0.325780 & 0.581526 & 0.189981 & $0.039^{*}$ \\
C12 & $0.36940(11)$ & $0.34394(7)$ & $0.1832(2)$ & $0.0156(3)$ \\
C13 & $0.29113(12)$ & $0.30435(8)$ & $0.1137(2)$ & $0.0191(3)$ \\
H13 & 0.229938 & 0.322934 & 0.071106 & $0.023^{*}$ \\
C14 & $0.30241(14)$ & $0.23966(8)$ & $0.1071(3)$ & $0.0236(3)$ \\
H14 & 0.249234 & 0.213740 & 0.061029 & $0.028^{*}$ \\
C15 & $0.39219(15)$ & $0.21232(7)$ & $0.1681(3)$ & $0.0261(4)$ \\
C16 & $0.46970(14)$ & $0.24787(8)$ & $0.2391(2)$ & $0.0243(3)$ \\
H16 & 0.530028 & 0.228129 & 0.281407 & $0.029^{*}$ \\
C17 & $0.45868(12)$ & $0.31356(7)$ & $0.2483(2)$ & $0.0191(3)$ \\
\hline
\end{tabular}

solid was obtained by vacuum filtration and washed with two portions of $2 \mathrm{~mL}$ of methanol. The crude product of $9.5450 \mathrm{~g}$ was then obtained. The crude $(1.0 \mathrm{~g})$ was transferred into two separate $100 \mathrm{~mL}$ narrow necked round-bottom flasks. In one flask, the crude was recrystallized from $40 \mathrm{~mL}$ of acetone and in the other flask the crude was recrystallized from a mixture of dichloromethane $(30 \mathrm{~mL})$ and methanol $(10 \mathrm{~mL})$. The orange needles of the title compound ssystematic name: 2,2'-(pentane-2,4-diylidene)bis(1(2,4-dinitrophenyl)hydrazine)\} had formed and were isolated by vacuum filtration.

M.p. 193 - 197 oC; IR: v( $\left.\mathrm{cm}^{-1}\right) 3329$ (s), $3108(\mathrm{~m}), 1615$ (vs), 1587 (vs), 1499 (vs), 1415 (s), 1360 (s), 1331 (vs), 1303 $(v s), 1269(v s), 1222(\mathrm{~m}), 1133(\mathrm{~s}), 1088(\mathrm{~s}), 1061(\mathrm{~s}), 916(\mathrm{~s})$, $831(\mathrm{~s}), 763(\mathrm{~m}), 726(\mathrm{~m}), 671(\mathrm{w}) .{ }^{1}$ H NMR: $(400 \mathrm{MHz}, \mathrm{d6}-$ DMSO): $\delta=11.13$ p.p.m. (s, 2H, NH), $9.14-9.13$ p.p.m. (d, $2 \mathrm{H}, \mathrm{J}=2.00 \mathrm{~Hz}$, aromatic H's), $8.34-8.31$ p.p.m. (dd, $2 \mathrm{H}$, $\mathrm{J}=9.5 \mathrm{~Hz}, 2.0 \mathrm{~Hz}$, aromatic H's), $7.94-7.91$ p.p.m. (d, 2H, $\mathrm{J}=9.5 \mathrm{~Hz}$, aromatic H's), 3.56 p.p.m. (s, $\left.2 \mathrm{H}, \mathrm{CH}_{2}\right) .2 .15$ p.p.m. (s, $\left.6 \mathrm{H}, \mathrm{CH}_{3}\right)^{13} \mathrm{C}$ NMR: $\delta=16.01$ p.p.m., 48.27 p.p.m., 116.30 p.p.m., 123.30 p.p.m., 130.20 p.p.m., 138.31 p.p.m., 144.93 p.p.m., 152.72 p.p.m.

\section{Experimental details}

Crystal evaluation and data collection were done on a Bruker Smart APEX2 diffractometer [1]. The structure was solved by the direct method using the SHELXS [2] program and refined. The visual crystal structure information was performed using ORTEP-3 [3] system software. All hydrogen atoms were placed in idealized positions and refined in riding models with $U_{\text {iso }}$ assigned the values of 1.2 times those of their parent atoms and the distances of $\mathrm{C}-\mathrm{H}$ were constrained to $0.95 \AA$ for all the aromatic $\mathrm{H}$ atoms, $0.98 \AA$ for methyl hydrogens, $0.99 \AA$ for methylene hydrogens and $0.88 \AA$ for imine hydrogens.

\section{Discussion}

The title compound is a polymorph of a previously reported $P \overline{1}$ structure [4] and a polymorph of a $P n$ structure [5]. The crystals are yellow in colour compared to the red blocks of the $P \overline{1}$ and $P n$ structures. The molecular overlay of the title compounds and the previously reported $P \overline{1}$ structure show an r.m.s. deviation of $1.5826 \AA$ while that of the title compound and the $P n$ structure is $0.5105 \AA$. In the former, the conformations of the two molecules are completely different while in the latter, the molecular conformations are similar. All three polymorphs enjoy intra molecular $\mathrm{N}-\mathrm{H} \cdots \mathrm{O}$ hydrogen bonds.

Acknowledgements: We acknowledge the University of KwaZulu-Natal and National Research Foundation of South Africa for financial support for Ekemini D. Akpan and Sizwe J. Zamisa.

\section{References}

1. Bruker. APEX3, SAINT-Plus, XPREP. Bruker AXS Inc., Madison, WI, USA (2016).

2. Sheldrick, G. M.: SHELXT - Integrated space-group and crystalstructure determination. Acta Crystallogr. A71 (2015) 3-8.

3. Farrugia, L. J.: WinGX and ORTEP for Windows. Appl. Crystallogr. 45 (2012) 849-854.

4. Naidu, S. M.; Krishnaiah, M.; Sivakumar, K.; Sharma, R. P.: 2,4Pentanedione bis(2,4-dinitrophenylhydrazone). Acta Crystallogr. Sect C 52 (1996) 1054.

5. Novruzova, A. B.; Kurbanova, M. M.; Magerramov, A. M.: 2,4Pentanedione bis(2,4-dinitrophenylhydrazone). Azerb. Khim. Zh. (Russ.)(Azerbaidzhan Chem. J.) 1 (2010) 21. 